\title{
Calcium/Calmodulin-Dependent Signaling for Prepenetration Development in Colletotrichum gloeosporioides
}

\author{
Kwang-Heum Uhm, Il-Pyung Ahn, Soonok Kim, and Yong-Hwan Lee
}

School of Agricultural Biotechnology, Seoul National University, Suwon 441-744, Korea.

Current address of I.-P. Ahn: Plant Biotechnology Division, National Institute of Agricultural Biotechnology, Rural Development Administration, Suwon 441-100, Korea.

Accepted for publication 12 August 2002.

\begin{abstract}
Uhm, K.-H., Ahn, I.-P., Kim, S., and Lee, Y.-H. 2003. Calcium/ calmodulin-dependent signaling for prepenetration development in Colletotrichum gloeosporioides. Phytopathology 93:82-87.

Colletotrichum gloeosporioides forms a specialized infection structure, an appressorium, for host infection. Contacting hard surface induces appressorium formation in $C$. gloeosporioides, whereas hydrophobicity of the contact surface does not affect this infection-related differentiation. To determine if the calcium/calmodulin-dependent signaling system is involved in prepenetration morphogenesis in C. gloeosporioides pathogenic on red pepper, effects of calcium chelator (EGTA), phospho-

zine, phenoxy benzamine, and W-7) were tested on conidial germination and appressorium formation. Exogenous addition of $\mathrm{Ca}^{2+}$, regardless of concentration, augmented conidial germination, while appressorial differentiation decreased at higher concentrations. Inhibition of appressorium formation by EGTA was partly restored by the addition of calcium ionophore $\mathrm{A} 23187$ or $\mathrm{CaCl}_{2}$. Calcium channel blockers and calmodulin antagonists specifically reduced appressorium formation at micromolar levels. These results suggest that biochemical processes controlled by the calcium/calmodulin signaling system are involved in the induction of prepenetration morphogenesis in C. gloeosporioides pathogenic on red pepper.
\end{abstract} lipase $\mathrm{C}$ inhibitor (neomycin), intracellular calcium modulators (TMB-8 and methoxy verampamil), and calmodulin antagonists (chloroproma-
Additional keywords: anthracnose, Glomerella cingulata.
Colletotrichum gloeosporioides (Penz.) Penz. \& Sacc., a homothallic Ascomycete, causes anthracnose diseases on more than 197 plant species including crops, vegetables, weeds, and trees (10). This fungus is also the causal agent of pepper anthracnose, one of the most devastating fruit diseases of red pepper (Capsicum annuиm L.) in Korea. For the infection to take place, a series of infection-related morphologenesis, initiated by spore adhesion to the host surface, spore germination, germ tube elongation, appressorium formation, and penetration by an infection peg, must precede. Previous studies with $C$. gloeosporioides and $C$. trifolii have shown that prepenetration morphogenesis, particularly appressorium formation, is induced by environmental cues involving thigmo-signals of the contact surface such as hydrophobicity and rigidity (34), and chemicals including host surface wax and ethylene (16).

These environmental signals, usually recognized by membranespanning receptors, are transferred into cells to express the appropriate cellular responses. The relaying of the signal is referred to as signal transduction and is mediated by second messengers including cyclic nucleotides $(18,19,31)$, calcium $(8,18)$, and inositol phosphate $(3,9)$.

Cytosolic calcium ion is regarded as an indispensable cation for cell growth and development and appressorial development in many organisms (13). The $\mathrm{Ca}^{2+}$ signaling pathway in cells is initiated via conformational changes of the GTP-binding protein (Gprotein) linked to a membrane receptor (17). Phospholipase $\mathrm{C}$ (PLC) is activated by the G-protein and hydrolyzes phophatidyl

Corresponding author: Y.-H. Lee; E-mail address: yonglee@plaza.snu.ac.kr

The first and second authors contributed equally.

Publication no. P-2002-1105-01R

(C) 2003 The American Phytopathological Society inositol-1,4-bisphosphate $\left(\mathrm{PIP}_{2}\right)$ into inositol-1,4,5-triphosphate $\left(\mathrm{IP}_{3}\right)$ and diacylglycerol (30). $\mathrm{IP}_{3}$ in turn causes the release of endogenous $\mathrm{Ca}^{2+}$ from intracellular calcium stores (1). Increments of intracellular $\mathrm{Ca}^{2+}$ result in the activation of calmodulin, one of the most ubiquitous and conserved $\mathrm{Ca}^{2+}$ receptor protein. $\mathrm{Ca}^{2+}$ binding induces conformational changes in calmodulin, resulting in the activation of many enzymes, including the multifunctional, dedicated calmodulin-dependent protein kinase and protein phosphatases $(15,32)$. Phenotypically, calmodulin mediates numerous fungal cell responses involving development (22), cytokinesis (20), and regulation of enzymatic activities (23).

In parallel, several reports suggest the involvement of the calcium/calmodulin signaling system of Colletotrichum spp. in the culmination of appressorium formation and disease occurrence over the past several years. Extensive studies using a wide range of pharmacological agents, which disrupt $\mathrm{Ca}^{2+}$ influxes or interfere with the calcium-binding protein, have strongly suggested the involvement of $\mathrm{Ca}^{2+}$ signaling systems in the appressorium formation of C. trifolii (34) and C. gloeosporioides pathogenic on avocado (15). More recently, calmodulin genes were cloned and characterized in these fungi. Calmodulin gene was highly expressed by hard-surface contact, ethylene, germination, and appressorium formation (15). Furthermore, reducing the functional transcript accumulation of this gene by expressing antisense copy resulted in the reduction of appressorium formation and perturbation of disease progress (35). These results suggest that calmodulin modulates the prepenetration events from spore attachment to appressorium formation in these fungi. However, no report has yet been made on the infection-related morphogenesis and related signaling system in C. gloeosporioides pathogenic on red pepper. Dissecting signaling pathways that transmit host surface-mimic signals into the cells might provide the new target sites for the development of effective control measures $(5,26)$.

In this study, we used several inhibitors thought to act on various steps in the calcium/calmodulin-dependent signaling 
pathway to elucidate the role of $\mathrm{Ca}^{2+} /$ calmodulin in the prepenetration morphogenesis of $C$. gloeosporioides. Our pharmacological data strongly indicate that the calcium/calmodulin-dependent signaling system is involved in the prepenetration development of C. gloeosporioides pathogenic on red pepper.

\section{MATERIALS AND METHODS}

Fungal isolate and cultural condition. $C$. gloeosporioides isolate virulent on pepper fruits was obtained from the National Institute of Agricultural Science and Technology, Rural Development Administration, Korea. Conidia were harvested from 7-dayold cultures grown on potato dextrose agar (PDA; Difco Laboratories, Detroit) at $25^{\circ} \mathrm{C}$ in the dark. Fungal stock was maintained as conidial suspension amended with $20 \%$ glycerol at $-70^{\circ} \mathrm{C}$ until use.

Conidial germination and appressorium formation. To induce conidial germination or appressorium formation, conidia were collected from PDA by mild scraping with sterile distilled water and washed twice by centrifugation. Fifty microliters of spore suspension $\left(1 \times 10^{5}\right.$ conidia per $\left.\mathrm{ml}\right)$ was placed onto hydrophobic and hydrophilic surfaces of GelBond (FMC Corp., Rockland, ME), microscopic coverslips (Marienfeld, Germany), and polystyrene petri dishes (GCEM Corp., Korea), which were then placed in a humid plastic box and incubated at room temperature for $12 \mathrm{~h}$. Conidia were considered germinated when germ tube length was equal to or greater than spore length. Appressorium was considered mature when melanization was evident. Percentages of germinated and germinating conidia to form appressoria were estimated from at least 100 conidia with three replicates per treatment. Experiments were repeated at least three times independently.

Chemical treatments. To test the effects of calcium-related chemicals, $45 \mu \mathrm{l}$ of conidial suspension $\left(1 \times 10^{5}\right.$ conidia per $\left.\mathrm{ml}\right)$ was placed on the hydrophobic side of GelBond and an additional $5 \mu \mathrm{l}$ of chemical solutions to be tested was added. Conidial germination and appressorium formation were measured on the substrata as described previously.

Preparation of chemicals. All chemicals used in this study were purchased from Calbiochem (La Jolla, CA) unless otherwise indicated. Tested chemicals were dissolved completely in proper solvents. EGTA (Sigma Chemical, St. Louis), a calcium chelator, was dissolved in distilled water and adjusted to $\mathrm{pH} 7.5$ with $10 \mathrm{~N}$ $\mathrm{NaOH}$. Neomycin, a PLC inhibitor, was dissolved in distilled

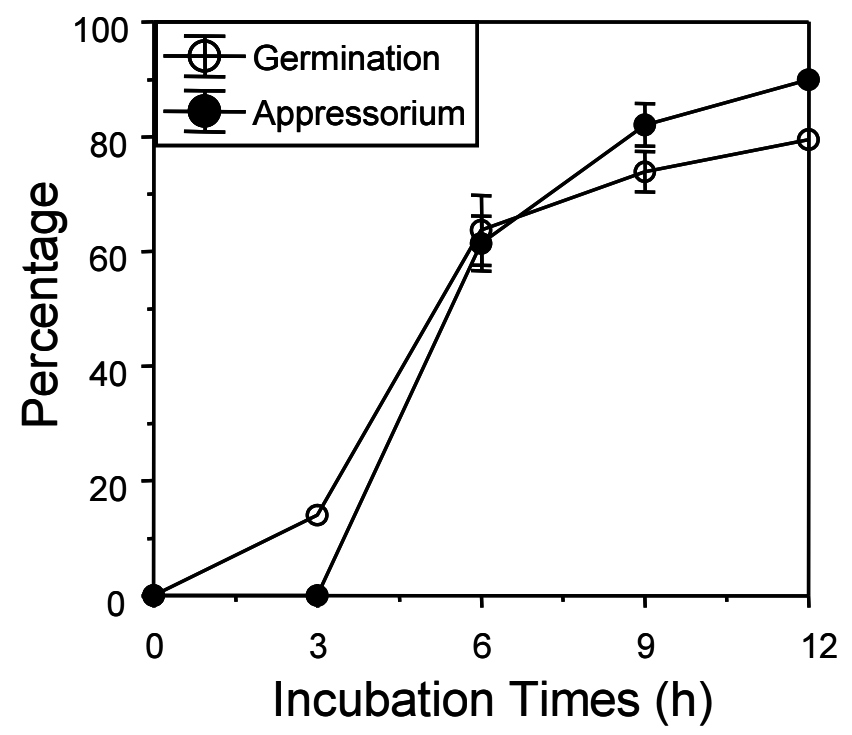

Fig. 1. Time course of conidial germination and appressorium formation of Colletotrichum gloeosporioides on the hydrophobic surface of GelBond. water. A23187, a calcium ionophore, and an intracellular calcium release inhibitor, TMB-8 [3,4,5-trimethoxybenzoic acid 8-(diethylamino)octyl ester], were dissolved in dimethyl sulfoxide (DMSO). Methoxy verampamil, a blocker of calcium channel anchored in the plasmamembrane, was dissolved in distilled water. Three calmodulin antagonists were prepared by dissolving W-7 [N-(6aminohexyl)-5-chloro-1-naphtalenesulfonamide], phenoxy benzamine, and chloropromazine in DMSO, ethanol, and distilled water, respectively.

\section{RESULTS}

Kinetics of conidial germination and appressorium formation. Conidia of C. gloeosporioides germinate only if surface contact is made. Following germination, when the germ tube encounters a hard surface, appressorium formation is initiated. In our observation, 2 to $3 \mathrm{~h}$ after contacting the hydrophobic surface of GelBond, conidia began to develop germ tubes (Fig. 1). More than 60 and $90 \%$ of the germinated conidia produced appressoria at 6 and $12 \mathrm{~h}$ after incubation, respectively. All hard surfaces tested induced appressorium development at high frequencies, including hydrophilic surface of GelBond (Table 1), whereas soft surfaces such as water agar (2\%) failed to induce appressorium formation (data not shown).

Effect of exogenous calcium, calcium chelator, and ionophore. To investigate the effect of exogenous $\mathrm{Ca}^{2+}$ concentration on appressorium formation and conidial germination, $\mathrm{CaCl}_{2}$ and EGTA were tested (Table 2; Fig. 2). Although addition of $\mathrm{CaCl}_{2}$ enhanced germination at the tested concentrations, 10 and $100 \mathrm{mM} \mathrm{CaCl} \mathrm{Cl}_{2}$ treatments inhibited appressorium formation by approximately $40 \%$ and to near abolishment, respectively. Addition of EGTA reduced conidial germination and appressorium formation in a dosage-dependent manner. The inhibition of appressorium formation by EGTA was partly restored by the addition of A23187 or $\mathrm{Ca}^{2+}$.

TABLE 1. Conidial germination and appressorium formation of Colletotrichum gloeosporioides on different substrata ${ }^{\mathrm{a}}$

\begin{tabular}{lcc}
\hline Substrata & $\begin{array}{c}\% \text { Conidial } \\
\text { germination }( \pm \mathrm{SD})\end{array}$ & $\begin{array}{c}\text { \% Appressorium } \\
\text { formation }( \pm \mathrm{SD})\end{array}$ \\
\hline GelBond (hydrophobic face) & $75.7 \pm 7.0$ & $98.3 \pm 2.1$ \\
GelBond (hydrophilic face) & $66.7 \pm 6.0$ & $81.7 \pm 6.7$ \\
Coverslips & $60.3 \pm 0.6$ & $94.7 \pm 2.5$ \\
Polystyrene & $69.0 \pm 4.0$ & $98.0 \pm 1.0$ \\
\hline
\end{tabular}

a Conidia of $C$. gloeosporioides were collected from potato dextrose agar, washed twice with distilled water, and placed on the hydrophobic surface of GelBond at a final concentration of $1 \times 10^{5}$ conidia per $\mathrm{ml}$.

TABLE 2. Effects of calcium chelator, ionophore, and $\mathrm{CaCl}_{2}$ on conidial germination and appressorium formation of Colletotrichum gloeosporioides ${ }^{\mathrm{a}}$

\begin{tabular}{cccc}
\hline Chemical & $\begin{array}{c}\text { \% Conidial } \\
\text { germination }( \pm \mathrm{SD})\end{array}$ & $\begin{array}{c}\text { \% Appressorium } \\
\text { formation }( \pm \mathrm{SD})\end{array}$ \\
\hline EGTA $(10 \mathrm{mM})$ & $15.7 \pm 2.1$ & $0.3 \pm 0.6$ \\
$(1 \mathrm{mM})$ & $46.3 \pm 2.5$ & $71.7 \pm 4.0$ \\
$(0.1 \mathrm{mM})$ & $67.0 \pm 1.7$ & $61.3 \pm 7.6$ \\
$\mathrm{CaCl}_{2}(100 \mathrm{mM})$ & $81.3 \pm 2.5$ & $1.7 \pm 0.6$ \\
$(10 \mathrm{mM})$ & $89.0 \pm 2.6$ & $54.7 \pm 2.3$ \\
$(1 \mathrm{mM})$ & $86.0 \pm 1.0$ & $88.3 \pm 1.5$ \\
EGTA $(10 \mathrm{mM})+\mathrm{A}_{23187}(10 \mu \mathrm{M})$ & $88.7 \pm 0.6$ & $88.0 \pm 1.0$ \\
EGTA $(10 \mathrm{mM})+\mathrm{CaCl}_{2}(1 \mathrm{mM})$ & $15.3 \pm 2.1$ & $40.0 \pm 10.0$ \\
Control & $59.6 \pm 2.6$ & $29.3 \pm 3.2$ \\
\hline
\end{tabular}

${ }^{\text {a }}$ Conidia of $C$. gloeosporioides were collected from potato dextrose agar, washed twice with distilled water, and placed on the hydrophobic surface of GelBond at a final concentration of $1 \times 10^{5}$ conidia per ml. Germination and appressorium development were determined microscopically at $12 \mathrm{~h}$ after incubation. 
Effect of PLC inhibitor. The phosphoinositide signaling system is related to calcium signaling through $\mathrm{IP}_{3}$, an intracellular calcium activator. $\mathrm{IP}_{3}$ is formed from $\mathrm{PIP}_{2}$ by activated PLC and released into the cytosol (9). To determine if phosphoinositide signaling participates in the appressorium formation, neomycin was tested (Fig. 3). Exogenous addition of neomycin, a PLC inhibitor, specifically inhibited appressorium formation by approximately $55 \%$ at $10 \mu \mathrm{M}$. However, germination was suppressed more severely at higher concentrations.

Effect of calcium channel modulators. Intracellular $\mathrm{Ca}^{2+}$ concentration may be regulated by the release of $\mathrm{Ca}^{2+}$ from intracellular stores or by $\mathrm{Ca}^{2+}$ channels located in the cell membrane
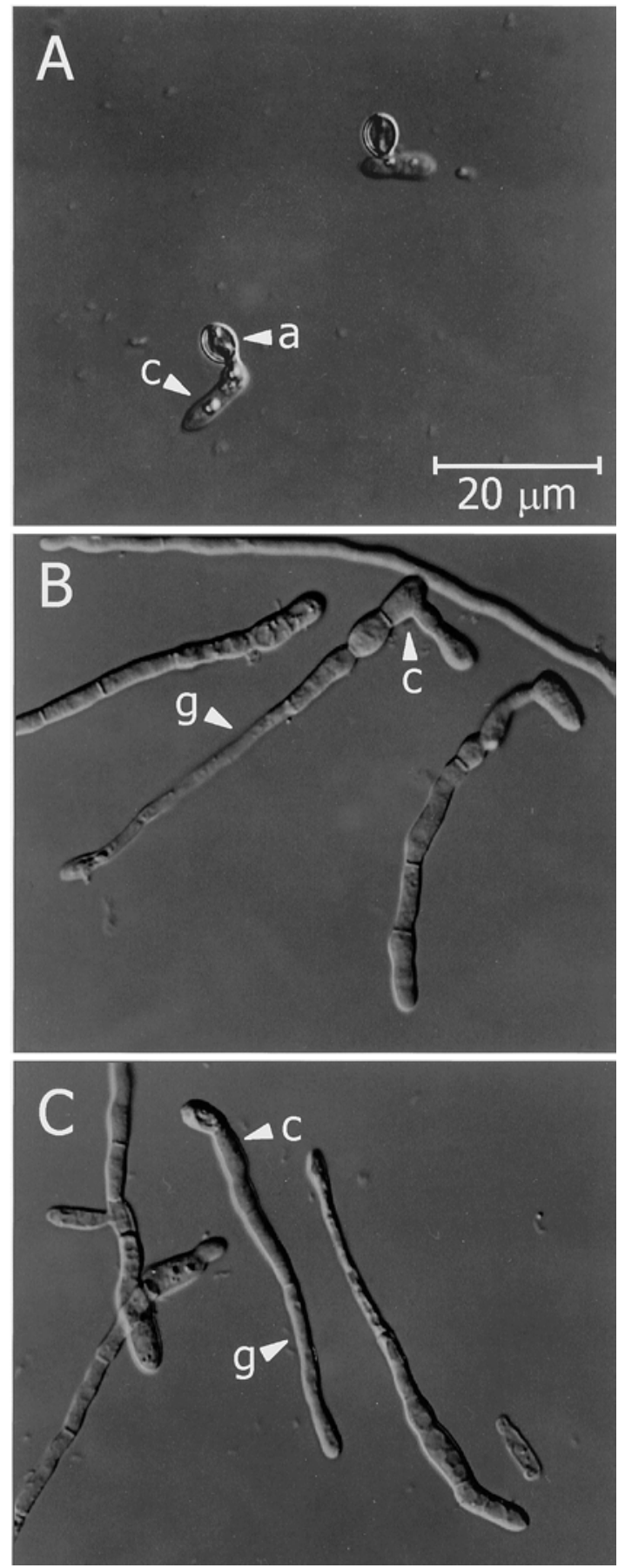

Fig. 2. Inhibition of appressorium formation of Colletotrichum gloeosporioides in the presence of $\mathbf{A}, \mathrm{H}_{2} \mathrm{O}, \mathbf{B}, 100 \mathrm{mM} \mathrm{CaCl}$, and $\mathbf{C}, 10 \mu \mathrm{M} \mathrm{W}-7$. Conidial suspension with chemicals was placed on the hydrophobic surface of GelBond and incubated in a moistened box at $25^{\circ} \mathrm{C}$ for $12 \mathrm{~h}$. a, appressorium; c, conidium; and g, germ tube.
(6). To determine if the modulation of calcium channels affects the conidial germination and appressorium formation of C. gloeosporioides, two calcium channel modulators were tested (Fig. 4). TMB-8, an inhibitor of calcium release from intracellular calcium store, specifically inhibited appressorium formation by 75 and $92 \%$ at 10 and $100 \mu \mathrm{M}$, respectively, but did not affect conidial germination. Methoxy verampamil, a calcium channel blocker located in the plasmamembrane, also specifically inhibited appressorium formation by $50 \%$ over a wide range of concentrations without affecting conidial germination.

Effect of calmodulin antagonists. The action of $\mathrm{Ca}^{2+}$ in cells is mainly mediated by calmodulin, the most potent $\mathrm{Ca}^{2+}$-binding protein. Calmodulin antagonists compete with $\mathrm{Ca}^{2+}$ for binding to calmodulin, and consequently inhibit $\mathrm{Ca}^{2+} /$ calmodulin signaling. To investigate whether calmodulin participates in the appressorium formation of $C$. gloeosporioides, the effects of several different antagonists were evaluated. All three calmodulin antagonists, chloropromazine, phenoxy benzamine, and W-7, have specific inhibitory effects on the appressorium formation (Figs. 2 and 5). Phenoxy benzamine and W-7 specifically inhibited appressorium formation, whereas conidial germination remained unaffected over a wide range of concentrations. Chloropromazine also selectively impaired appressorium formation at 1 and $10 \mu \mathrm{M}$ concentrations; however, conidial germination and appressorium formation were both inhibited at $100 \mu \mathrm{M}$.

\section{DISCUSSION}

Extensive research has been conducted to identify environmental cues and cellular signaling systems in the infection-related morphogenesis of plant pathogenic fungi during the last decade. Accumulated data suggested that physicochemical signals, including rigidity and hydrophobicity of the contact surface and host surface chemicals, are the determinants inducing infection structure formation in several plant pathogenic fungi $(12,19,34)$. In this study, however, C. gloeosporioides pathogenic on red pepper required rigidity, not hydrophobicity of the contact surface, for the induction of appressorium formation. Similar results have been described in the appressorium formation of $C$. graminicola (4). On the other hand, hydrophobicity of the contact surface is one of the determining signals for appressorium formation in C. trifolii (34) and a well-studied rice blast fungus, Magnaporthe grisea (19).

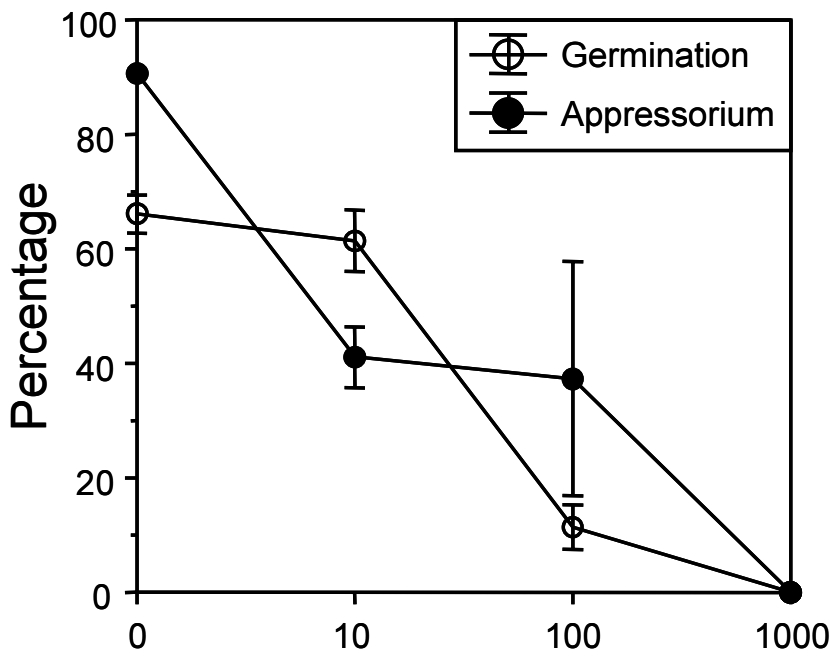

Neomycin Concentration $(\mu \mathrm{M})$

Fig. 3. Effects of neomycin on conidial germination and appressorium formation in Colletotrichum gloeosporioides. Conidial suspensions with different concentrations of neomycin were placed on the hydrophobic surface of GelBond and incubated in a moistened box at $25^{\circ} \mathrm{C}$ for $12 \mathrm{~h}$. 
These variances suggest the possibility of different mechanisms in pathogenesis including host-sensing among different species of Colletotrichum as well as other plant pathogenic fungi.

Addition of calcium selectively inhibited appressorium formation at higher concentrations. In addition, treatment of calcium chelator (EGTA) inhibited appressorium formation and conidial germination. These results indicate that maintaining the level of intracellular $\mathrm{Ca}^{2+}$ concentration is important for appressorium formation in C. gloeosporioides. This is further supported by the fact that EGTA inhibition of appressorium formation was partly restored by the addition of exogenous calcium or calcium ionophore (A23187). Taken together, these results strongly suggest that sustained calcium homeostasis, rather than transient change of $\mathrm{Ca}^{2+}$, in the cytosol is important for appressorium formation in this fungus. Similar phenomena have been suggested for appressorium formation in $C$. trifolii (34) and other fungi such as Zoophthora radicans (24), Metarhizium anisopliae (29), and Phyllosticta ampelicida (27). Treatment with neomycin, a PLC inhibitor, resulted in the reduction of appressorium formation in $C$.
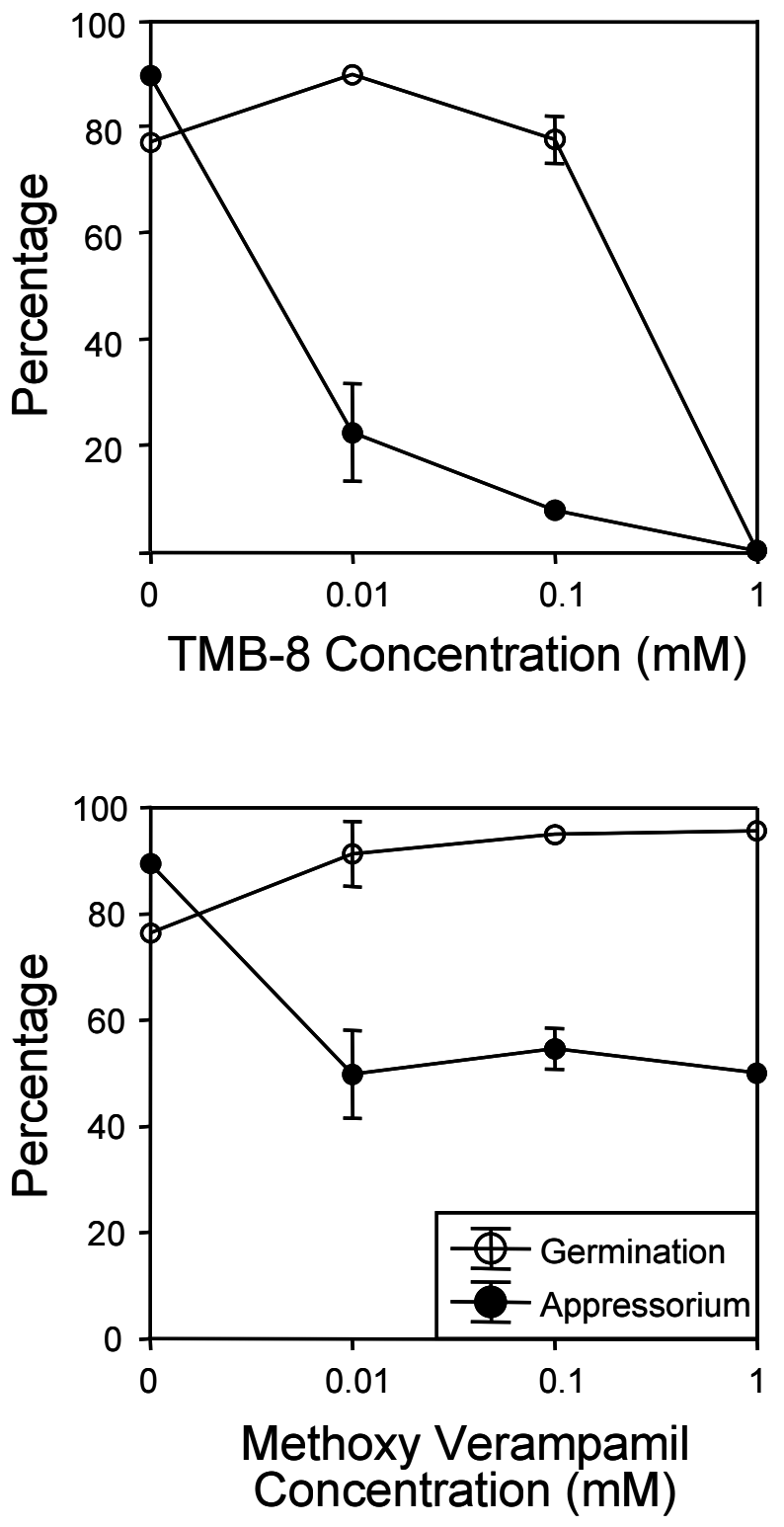

Fig. 4. Effects of calcium channel modulators on conidial germination and appressorium formation in Colletotrichum gloeosporioides. Conidial suspensions with different concentrations of chemicals were placed on the hydrophobic surface of GelBond and incubated in a moistened box at $25^{\circ} \mathrm{C}$ for $12 \mathrm{~h}$.
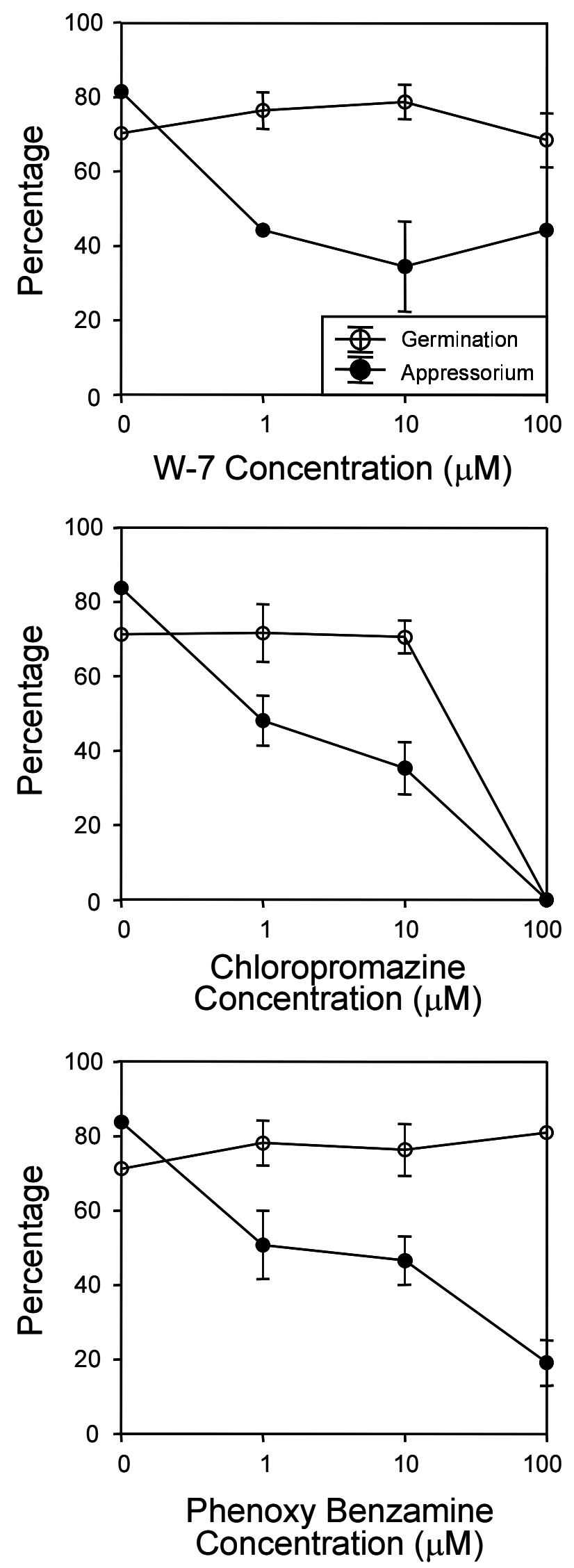

Fig. 5. Effects of calmodulin antagonists on conidial germination and appressorium formation in Colletotrichum gloeosporioides. Conidial suspensions with different concentrations of chemicals were placed on the hydrophobic surface of GelBond and incubated in a moistened box at $25^{\circ} \mathrm{C}$ for $12 \mathrm{~h}$. 
gloeosporioides. Release of calcium from intracellular calcium stores by $\mathrm{IP}_{3}$ is indispensable for the induction of appressorium formation. To understand the possible involvement of this mechanism more directly, the effects of calcium channel modulator on conidial germination and appressorium formation of C. gloeosporioides were tested. Both calcium channel modulators, TMB-8 and methoxy verampamil, specifically reduced appressorium formation over a wide range of concentrations, further supporting the results that maintenance of cytosolic $\mathrm{Ca}^{2+}$ within a certain range is indispensable for appressorium formation in this fungus. In addition, greater reduction of appressorium formation was induced by TMB-8 than by methoxy verampamil. This indicates that $\mathrm{Ca}^{2+}$ release from internal calcium stores is more important than $\mathrm{Ca}^{2+}$ influx mediated through the plasmamembrane gate on the appressorium formation. Similar results were observed in our previous study on appressorium formation of Magnaporthe grisea (18). However, both conidial germination and appressorium formation were inhibited without selectivity in Phyllosticta ampelicida (27) and $C$. trifolii (34). It should be noted that TMB-8 affects not only $\mathrm{Ca}^{2+}$ channels but other signal transduction pathways such as protein kinase C (28). Therefore, the results should be interpreted cautiously.

Although inhibition of prepenetration morphogenesis by the calmodulin antagonists has been described previously in the filamentous fungi, notable differences exist in their inhibitory patterns. All three calmodulin antagonists specifically inhibited appressorium formation at micromolar levels in this study, whereas conidial germination remained unaffected. Specific inhibition of appressorium formation by calmodulin antagonists was reported in C. trifolii (34) and Magnaporthe grisea (18). On the other hand, nonspecific inhibitions of conidial germination and appressorium formation were reported in Phyllosticta ampelicida (27). These variable inhibitory effects on appressorium formation among the fungal species might be due to the alternatives of biochemical signaling pathways via calmodulin rather than to the structural differences of calmodulin in these fungi. This is supported by the fact that calmodulin genes are highly conserved and nearly identical in their nucleotide sequences in several fungi $(15,21,35)$.

Comparative analyses on $\mathrm{Ca}^{2+}$ signaling for appressorium formation between $C$. gloeosporioides isolates pathogenic on red pepper in the current study and on avocado unveiled significantly different responses against calmodulin antagonists. Specific inhibition on appressorium formation was observed in a red pepper isolate, whereas nonspecific inhibition on conidial germination and appressorium formation was observed in an avocado isolate (15). Furthermore, in C. gloeosporioides pathogenic on avocado, treatment of a PLC inhibitor, U73122, resulted in the synchronous inhibition of conidial germination and appressorium formation at $64 \mathrm{nM}$. However, selective inhibition of appressorium formation by another PLC inhibitor, neomycin, was observed at $10 \mu \mathrm{M}$ in this study. Although different inhibitory effects were observed through treatments of PLC inhibitors, the validity of these effects remains unclear because different chemicals (U73122 and neomycin) were used in each experiment. In addition, appressorium formation of $C$. gloeosporioides pathogenic on avocado was selectively triggered by the surface wax of its host avocado, but not by other plant surface waxes. However, appressorium formation of $C$. gloeosporioides pathogenic on red pepper in the current study did not show such host-specific responses. Treatments of plant waxes from red pepper fruits and leaves, leaves of cabbage (Brassica oleracea), and Japanese ivy (Parthenocissus tricuspidata) enhanced appressorium formation (data not shown). Taken together, these results suggest that differences in signaling for appressorium formation exist between $C$. gloeosporioides pathogenic on avocado and on red pepper. This speculation may be further supported by the complexity on the taxonomy of $C$. gloeosporioides. von Arx (33) subsumed 594 species into a large one, $C$. gloeosporioides, based on the morphological characteristics. As a result, this species is composed of not only mycologically but genetically and pathogenically diverse strains $(2,11)$. This complexity has also been demonstrated in the vegetative compatibility grouping and DNA fingerprinting analyses of this fungus $(7,14,25)$. High level of species heterogeneity might induce strain-specific responses in the signaling cascades regulating the prepenetration morphogenesis in $C$. gloeosporioides.

In conclusion, maintaining calcium homeostasis in cells is important for appressorium formation in C. gloeosporioides pathogenic on red pepper. Calcium homeostasis in the cytosol appears to be controlled by complex biochemical mechanisms including calcium channels in the cell membrane, cytosolic stores, and binding with calmodulin. Although the precise mechanisms of signaling pathways are still unclear, we demonstrate that the calcium/calmodulin-dependent signaling system is involved in the appressorium formation of $C$. gloeosporioides pathogenic on red pepper.

\section{ACKNOWLEDGMENTS}

This research was supported by a grant (PF002110-02) from the Plant Diversity Research Center of 21st Century Frontier Research Program funded by Ministry of Science and Technology of the Korean government and a grant from the Agricultural Plant Stress Research Center funded by Korea Science and Engineering Foundation to Y.-H. Lee. S. Kim is grateful for the postdoctoral fellowship provided by the Ministry of Education through the Brain Korea 21 Project.

\section{LITERATURE CITED}

1. Belde, P. J., Vossen, J. H., Borst-Pauwels, G. W., and Theuvenet, A. P. 1993. Inositol 1,4,5-trisphosphate releases $\mathrm{Ca}^{2+}$ from vacuolar membrane vesicles of Saccharomyces cerevisiae. FEBS Lett. 323:113-118.

2. Bernstein, B., Zehr, E. I., Dean, R. A., and Shabi, E. 1995. Characteristics of Colletotrichum from peach, apple, pecan, and other hosts. Plant Dis. 79:478-482.

3. Berridge, M. J. 1993. Inositol triphosphate and calcium signalling. Nature 361:315-325.

4. Chaky, J., Anderson, K., Moss, M., and Vaillancourt, L. 2001. Surface hydrophobicity and surface rigidity induce spore germination in Colletotrichum graminicola. Phytopathology 91:558-564.

5. Choi, W.-B., Kang, S.-H., Lee, Y.-W., and Lee, Y.-H. 1998. Cyclic AMP restores appressorium formation inhibited by polyamines in Magnaporthe grisea. Phytopathology 88:58-62.

6. Cornelius, G., Gebauer, G., and Techel, D. 1989. Inositol triphosphate induces calcium release from Neurospora crassa vacuoles. Biochem. Biophys. Res. Commun. 162:852-856.

7. Correll, J. C., Weidemann, G. J., TeBeest, D. O., and Guerber, J. C. 1991. Genetic and molecular diversity in Colletotrichum gloeosporioides. (Abstr.) Phytopathology 81:1219.

8. David, E. C. 1995. Calcium signaling. Cell 47:423-431.

9. Devecha, N., and Irvine, R. F. 1995. Phospholipid signaling. Cell 80:269-278.

10. Farr, D., Bills, G. F., Chamuris, G. P., and Rossman, A. Y. 1989. Fungi on Plants and Plant Products in the United States. The American Phytopathological Society, St. Paul, MN.

11. Freeman, S., and Shabi, E. 1996. Cross-infection of subtropical and temperate fruits by Colletotrichum species from various hosts. Physiol. Mol. Plant Pathol. 49:395-404.

12. Hwang, C. S., and Kolattukudy, P. E. 1995. Isolation and characterization of genes expressed uniquely during appressorium formation by Colletotrichum gloeosporioides conidia induced by the host surface wax. Mol. Gen. Genet. 247:282-294.

13. Jackson, S. L., and Heath, I. B. 1993. Roles of calcium ions in hyphal tip growth. Microbiol. Rev. 57:367-382.

14. Kelemu, S., Skinner, D. Z., Badel, J. L., Moreno, C. X., Rodriguez, M. X., Fernandes, C. D., Charchar, M. J., and Chakraborty, S. 1999. Genetic diversity in South American Colletotrichum gloeosporioides isolates from Stylosanthes guianensis, a tropical forage legume. Eur. J. Plant Pathol. 105:261-272.

15. Kim, Y. K., Li, D., and Kolattukudy, P. E. 1998. Induction of $\mathrm{Ca}^{2+}-$ calmodulin signaling by hard-surface contact primes Colletotrichum gloeosporioides conidia to germinate and form appressoria. J. Bacteriol. 180:5144-5150. 
16. Kolattukudy, P. E., Rogers, L. M., Li, D., Hwang, C. S., and Flaishman, M. A. 1995. Surface signaling in pathogenesis. Proc. Natl. Acad. Sci. USA 92:4080-4087.

17. Larson, T. G., Choi, G. H., and Nuss, D. 1992. Regulatory pathways governing modulation of fungal gene expression by a virulence-attenuating mycovirus. EMBO J. 11:4539-4548.

18. Lee, S.-C., and Lee, Y.-H. 1998. Calcium/calmodulin-dependent signaling for appressorium formation in the plant pathogenic fungus Magnaporthe grisea. Mol. Cells 8:698-704.

19. Lee, Y.-H., and Dean, R. A. 1994. Hydrophobicity of contact surface induces appressorium formation in Magnaporthe grisea. FEMS Microbiol. Lett. 115:71-76.

20. Liu, T., Williams, J. G., and Clarke, M. 1992. Inducible expression of calmodulin antisense RNA in Dictyostelium cells inhibits the completion of cytokinesis. Mol. Biol. Cell 3:1403-1413.

21. Liu, Z. M., and Kolattukudy, P. E. 1999. Early expression of the calmodulin gene, which precedes appressorium formation in Magnaporthe grisea, is inhibited by self-inhibitors and requires surface attachment. J. Bacteriol. 181:3571-3577.

22. Lydan, M. A., and O'Day, D. H. 1988. Different developmental functions for calmodulin in Dictyostelium: Trifluoperazine and R24571 both inhibit cell and pronuclear fusion but enhance gamete formation. Exp. Cell Res. 178:51-63.

23. Mach, R. L., Zeilinger, S., Kristufek, D., and Kubicek, C. P. 1998. $\mathrm{Ca}^{2+-}$ calmodulin antagonists interfere with xylanase formation and secretion in Trichoderma reesei. Biochem. Biophys. Acta 1403:281-289.

24. Magalhaes, B. P., Wayne, R., Haumber, R. A., Shields, E. J., and Roberts, D. W. 1991. Calcium-regulated appressorium formation of the entomopathogenic fungus Zoophthora radicans. Protoplasma 160:77-88.

25. Mills, P. R., Sreenivasaprasad, S., and Brown, A. E. 1992. Detection and differentiation of Colletotrichum gloeosporioides isolates using PCR. FEMS Microbiol. Lett. 77:137-143.
26. Oh, H.-S., and Lee, Y.-H. 2000. A target-site-specific screening system for antifungal compounds on appressorium formation in Magnaporthe grisea. Phytopathology 90:1162-1168.

27. Shaw, B. D., and Hoch, H. C. 2000. $\mathrm{Ca}^{2+}$ regulation of Phyllosticta ampelicida pycnidiospore germination and appressorium formation. Fungal Genet. Biol. 31:43-53.

28. Simpson, A. W. M., Hallman, T. J., and Rink, T. J. 1984. TMB-8 inhibits secretion evoked by phorbol ester at basal cytoplasmic free calcium in quin-2-loaded platelets much more effectively than it inhibits thrombininduced mobilization. FEBS Lett. 176:139-143.

29. St. Leger, R. J., Roberts, D. W., and Staples, R. C. 1991. A model to explain differentiation of appressoria by germlings of Metarhizium anisopliae. J. Invertebrate Pathol. 57:299-310.

30. Su, Z., Csutora, P., Hunton, D., Shoemaker, R. L., Marchase, R. B., and Blalock, J. E. 2001. A store-operated nonselective cation channel in lymphocytes is activated directly by $\mathrm{Ca}^{2+}$ influx factor and diacylglycerol. Am. J. Physiol. Cell Physiol. 280:C1284-C1292.

31. Takano, Y., Komeda, K., Kojima, K., and Okuno, T. 2001. Proper regulation of cyclic AMP-dependent protein kinase is required for growth, conidiation, and appressorium function in the anthracnose fungus Colletotrichum lagenarium. Mol. Plant-Microbe Interact. 14:1149-1157.

32. Van Edick, C. G., and Waterson, D. M. 1995. Calmodulin structure and function. Pages 105-126 in: Calcium and Cell Physiology. D. Marme, ed. Springer-Verlag, Berlin.

33. von Arx, J. A. 1957. Die arten der Gattung Colletotrichum cda. Phytopathol. Z. 29:413-468.

34. Warwar, V., and Dickman, M. B. 1996. Effects of calcium and calmodulin on spore germination and appressorium development in Colletotrichum trifolii. Appl. Environ. Microbiol. 62:74-79.

35. Warwar, V., Oved, S., and Dickman, M. B. 2000. Antisense expression of the calmodulin gene from Colletotrichum trifolii impairs prepenetration development. FEMS Microbiol. Lett. 191:213-219. 